\title{
Importance of genetic drift during Pleistocene divergence as revealed by analyses of genomic variation
}

\author{
L. LACEY KNOWLES and CORINNE L. RICHARDS \\ Department of Ecology and Evolutionary Biology, University of Michigan, Ann Arbor, Michigan 48109-1079, USA
}

\begin{abstract}
Determining what factors affect the structuring of genetic variation is key to deciphering the relative roles of different evolutionary processes in species differentiation. Such information is especially critical to understanding how the frequent shifts and fragmentation of species distributions during the Pleistocene translates into species differences, and why the effect of such rapid climate change on patterns of species diversity varies among taxa. Studies of mitochondrial DNA (mtDNA) have detected significant population structure in many species, including those directly impacted by the glacial cycles. Yet, understanding the ultimate consequence of such structure, as it relates to how species divergence occurs, requires demonstration that such patterns are also shared with genomic patterns of differentiation. Here we present analyses of amplified fragment length polymorphisms (AFLPs) in the montane grasshopper Melanoplus oregonensis to assess the evolutionary significance of past demographic events and associated drift-induced divergence as inferred from mtDNA. As an inhabitant of the sky islands of the northern Rocky Mountains, this species was subject to repeated and frequent shifts in species distribution in response to the many glacial cycles. Nevertheless, significant genetic structuring of $M$. oregonensis is evident at two different geographic and temporal scales: recent divergence associated with the recolonization of the montane meadows in individual sky islands, as well as older divergence associated with displacements into regional glacial refugia. The genomic analyses indicate that drift-induced divergence, despite the lack of long-standing geographic barriers, has significantly contributed to species divergence during the Pleistocene. Moreover, the finding that divergence associated with past demographic events involves the repartitioning of ancestral variation without significant reductions of genomic diversity has intriguing implications - namely, the further amplification of drift-induced divergence by selection.
\end{abstract}

Keywords: AFLPs, coalescence, genomic, historical demography, sky islands, speciation, statistical phylogeography

Received 26 May 2005; revision accepted 25 July 2005

\section{Introduction}

The Pleistocene glaciations indisputably had a major impact on many taxa as species distributions shifted repeatedly and frequently in response to the climatic fluctuations (Huntley \& Webb 1989; Pielou 1991; Webb \& Bartlein 1992; Joseph et al. 1995; Green et al. 1996; Hewitt 1996; Roy et al. 1996); but how these effects translate into population or

Correspondence: L. Lacey Knowles, Fax: 734-763-4080; E-mail: knowlesl@umich.edu species divergence is not clear. Based on analyses of the timing of species origin, it is now clear that the effects of the Pleistocene glaciations varied among taxa (e.g. Weir \& Schluter 2004), apparently inhibiting divergence in some species (e.g. Cracraft \& Prum 1988; Bush 1994; Riddle 1996) but not others (e.g. Bermingham et al. 1992; Klicka \& Zink 1997; Avise \& Walker 1998; Knowles \& Otte 2000; Knowles 2000, 2001a; Ayoub \& Riechert 2004). One step toward understanding the differing response to this dynamic history is to understand what factors contribute to species divergence. Two hypotheses might explain how species were able to diversify during the dynamic Pleistocene: 
(i) divergence is selectively driven, or (ii) genetic drift associated with displacements into glacial refugia and /or recolonization of previously glaciated areas has promoted divergence, thereby implying that the glacial cycles themselves played a direct role in Pleistocene speciation.

The genetic signature of past biogeographic and demographic events has been studied primarily from mitochondrial DNA (mtDNA) sequences. The smaller effective population size of $\mathrm{mtDNA}$ as compared to nuclear loci makes mtDNA a desirable molecular marker for reconstructing detailed species histories, especially with the shift towards analysing such data in a statistical-phylogeographic framework (Knowles \& Maddison 2002; Knowles 2004). However, consideration of many loci is essential if the evolutionary significance of past demographic and biogeographic events (in terms of their contribution to species divergence) is to be determined. Individual loci can evolve under a diverse set of models, including selection. Therefore, only with an analysis of genomic variation can the base-level effect of genetic drift be assessed (e.g. Latta et al. 2001). For example, significant population structure revealed by analysis of mtDNA may suggest drift-induced changes played a prominent role in species divergence. However, the evolutionary significance of such events is limited by the extent to which patterns of genomic variation have been similarly affected.

Here we analyse patterns of genomic variation in the montane grasshopper Melanoplus oregonensis to assess the evolutionary significance of past demographic events associated with the repeated shifts in species distributions during the Pleistocene. $M$. oregonensis is among a group of Rocky Mountain sky island inhabitants that originated during the Pleistocene and underwent a rapid radiation (i.e. 38 species within the last 2 million years) (Knowles 2000; Knowles \& Otte 2000). M. oregonensis is flightless with extremely reduced wings and broadly distributed among the 'sky islands' of the northern Rocky Mountains. This region was covered by the Cordilleran ice sheet and a network of mountain and valley glaciers (Hollin \& Schilling 1981; Mayewski et al. 1981; Pielou 1991). Palynological records indicate that taxa in this region underwent both latitudinal and elevational shifts in distribution repeatedly in response to the many glacial cycles (Wolfe 1987; Singh 1988; Huntley \& Webb 1989; Pielou 1991; Elias 1996).

Despite this dynamic history, statistical-phylogeographic analysis of mtDNA sequence variation in $M$. oregonensis suggests that divergence in this species occurred at two different temporal and spatial scales (Knowles 2001a, b). Recent and local divergence associated with the recolonization of individual sky islands, as well as regional differences associated with displacements into allopatric glacial refugia in the past, were inferred from patterns of mtDNA variation. Drift, rather than gene flow, appears to be the dominant force structuring genetic variation in M. oregonensis.
Genetic variation in M. oregonensis is highly structured, as indicated by significant $F_{\mathrm{ST}}$ analyses and genealogical estimates (Knowles 2001b). None of the mitochondrial haplotypes are geographically widespread and all shared haplotypes occurred between phylogenetically closely related populations, not geographically proximate populations, indicating a lack of current gene flow or isolation by distance. Similarly, there is not a relationship between $F_{\mathrm{ST}}$ values and the geographic distances separating populations (see Fig. 7, Knowles 2001b). Instead, there is substantial variation among the $F_{\mathrm{ST}}$ values of individual pairs of populations across all geographic distances, which is consistent with a drift-dominated system (Hutchinson \& Templeton 2000). This is in accord with the natural history of the grassshoppers (i.e. M. oregonensis is flightless and restricted to isolated montane meadows).

Significant genetic structure and differentiation is not expected in genomic patterns of variation unless the driftinduced divergence associated with past demographic events has indeed played a critical role in Pleistocene divergence - species distributions shifted frequently in response to the glacial cycles and nuclear loci require a greater amount of time for lineage sorting compared to mtDNA. Here we characterize and analyse AFLP data to test the hypothesis that both recent and local, as well as older and regional, demographic events have played a significant role in species divergence (i.e. patterns of genomic variation reflect divergence associated with two different spatial and temporal scales). Using an explicit geographic framework, we are able to demonstrate that despite a dynamic history, significant genomic differentiation is associated with both displacements into glacial refugia and recolonization of previously glaciated areas. These results provide strong support for Pleistocene divergence models postulating that shifting species distributions would have promoted species divergence (e.g. Mengel 1964; Haffer 1969, 1997; Hewitt 1996, 1999). Rather than relegating genetic drift as an insignificant factor in species divergence (e.g. Coyne \& Orr 2004), this study demonstrates the evolutionary importance of drift-induced divergence associated with past demographic events, even in the absence of long-term biogeographic barriers.

\section{Materials and methods}

\section{Collections, DNA sequencing, and AFLPS}

Specimens were collected throughout the range of Melanoplus oregonensis (Acrididae: Melanoplinae: Indigens species group) (Knowles 2000) from the sky islands of mountain ranges in western Montana and northwestern Wyoming (Fig. 1). Populations throughout its range were sampled; 134 individuals from 14 populations were collected (see Appendix). 


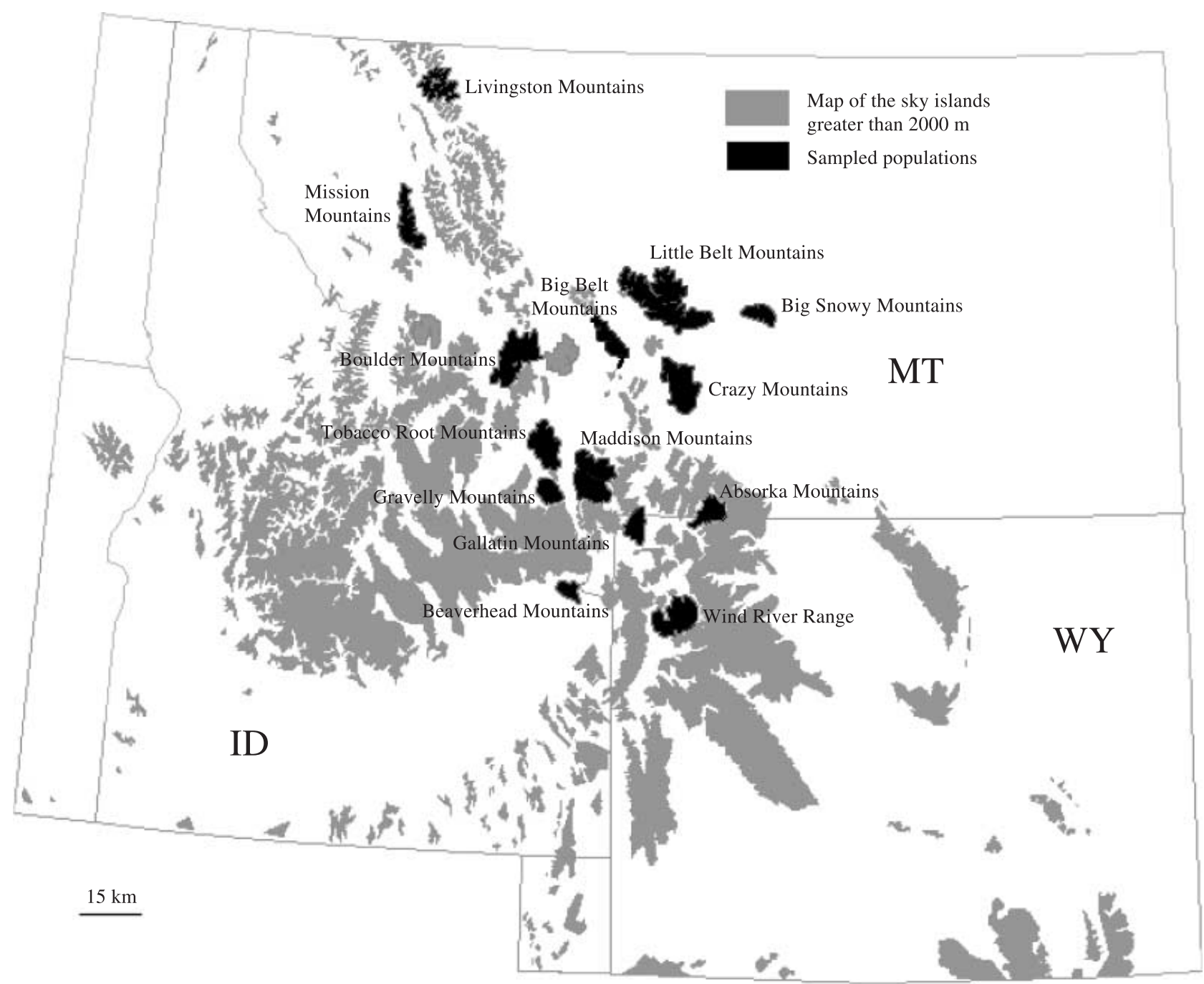

Fig. 1 Map of the sky islands of northern Rocky Mountains showing the geographic distribution of sampled populations (ID, Idaho; MT, Montana; WY, Wyoming).

AFLP fragments generated by 16 primer pair combinations (E-ACC and E-ACT each paired with M-CAA, CAC, CAG, CAT, CTA, CTC, CTG, and CTT, where E is EcoRI and $\mathrm{M}$ is $\mathrm{Mse} \mathrm{I}$ ) were used in a preliminary screen of 12 individuals from different populations. Based on these results, two primer combinations (E-ACC/M-CTT and E-ACT/M-CTT) were chosen because they produced a manageable number of fragments that were well separated. AFLP fingerprints were generated with the 'AFLP analysis system 1' according to the kit protocol (Invitrogen). An EcoRI selective primer labelled with fluorescent dye (FAM, blue) was used for runs on an $\mathrm{ABI} 3730$ sequencer with the internal size standard ROX500 (Applied Biosystems). To minimize the extent of size homoplasy among AFLP markers (Vekemans et al. 2002), small fragment sizes were avoided and target fragments (i.e. distinct bands that display the same electro- phoretic mobility) were chosen within the size range of 90-550 base pairs (e.g. Wang et al. 2003). Each individual was analysed for the presence or absence of AFLP products using GENOGRAPHER software (version 1.6, Montana State University; http://hordeum.msu.montana.edu/ genographer). Only unambiguously detectable fragments were scored.

Because the AFLP method employs a polymerase chain reaction (PCR), the technique can rapidly generate large numbers of reproducible anonymous markers for organisms in which there is a lack of prior genomic information. Consequently, AFLPs have been widely used as genetic markers. Moreover, they have a demonstrated utility for various types of study due to their random genomic distribution, including applications for phylogeographic (e.g. Mueller \& Wolfenbarger 1999; Wang et al. 2003; Carisio 
et al. 2004) and mapping studies [quantitative trait loci (QTL) analyses in particular; e.g. Kocher et al. 1998; Hawthorne 2001; Parsons \& Shaw 2002]. To confirm the reliability of AFLPs in this study, a subset of 20 loci was also electrophoretically separated on a manual gel, where AFLP bands were visualized with silver staining, excised (see Vos et al. 1995 for protocol), and sequenced (e.g. Parsons \& Shaw 2002). Likewise, two separate amplifications for independent determination of individual genotypes were examined for a subset of individuals $(n=48)$ to confirm the repeatability of the AFLP procedure (error rate $=0.04 \%$ per locus). An interlocus comparison was also used to confirm that divergence of individual loci was consistent with a neutral model (using the program FDIST, Beaumont \& Nichols 1996; contrasts were based on comparison of M. oregonensis to Melanoplus marshalli, Knowles unpublished).

\section{Data analysis}

A Bayesian framework was first used to determine whether the geographic model inferred from the mtDNA gene tree (Knowles 2001b) accurately reflects the species history. To identify ancestral glacial refugial source populations, the population structure was inferred as a set of $k$ clusters delimited by distinct allele frequencies based on the AFLP data. The posterior probability conditioned on priors based on the presumed refuges inferred from the mtDNA phylogeny (see Appendix), that is an eastern, central, and western glacial refugial source population (Knowles 2001a), were estimated with the model-based clustering method in STRUCTURE (Pritchard et al. 2000). Individuals were assigned probabilistically to the $k$ inferred glacial refugial source populations. A burn-in period of 30000 iterations was used and data were collected for $10^{6}$ iterations under a noadmixture model for each of three replicates to test for a correspondence between the mtDNA-based geographic model and patterns of genomic variation.

Estimates of the genetic diversity and genetic structure at both the local and regional geographic scales were then tested using moment and likelihood estimators. Hierarchical structuring of genetic variation (i.e. regional differences associated with displacements into glacial refugia and local divergence associated with individual sky islands, see Appendix) and pairwise $\Phi_{\mathrm{ST}}$ distances were measured using an analysis of molecular variance (AMOVA) (ARLEQUIN 1.1, Excoffier et al. 1992); an assumption of Hardy-Weinberg equilibrium was used for these calculations since AFLP markers must be analysed as dominant loci. Significance of the variance components calculated from the AMOvA was determined with 1000 permutations. An analogous measure of the $F_{\mathrm{ST}}$ approach of Hudson et al. (1992), $\theta^{B}$, and Nei's gene diversity (1987), $H_{S}$, were also calculated using a Bayesian approach that incorporates uncertainty about Hardy-Weinberg proportions (HICKORY, Holsinger et al.
Table 1 Inferring the number of clusters, $k$, with STRUCTURE program* (a model-based Bayesian clustering method) without using population priority information (burn-in of 30000 iterations and collected data for $10^{6}$ iterations; 3 independent simulations were run to establish posterior probabilities of $k$ were consistent

\begin{tabular}{lll}
\hline$k$ & $\ln P(x \mid k)$ & $P(k \mid x)$ \\
\hline 1 & -6955.4 & $\sim 0$ \\
2 & -6445.2 & $\sim 0$ \\
3 & -6161.7 & $\sim 1$ \\
4 & -6666.5 & $\sim 0$ \\
5 & -6791.7 & $\sim 0$ \\
6 & -6866.1 & $\sim 0$ \\
\hline
\end{tabular}

*The estimated posterior probabilities are indicative of which models are consistent with the data (Pritchard et al. 2000).

2002); a free model and a burn-in of 50000 and sampling runs of 500000 iterations were used in each of three replicate runs.

\section{Results}

A total of 140 AFLP loci were scored in each individual; none were fixed within specific populations. The posterior probabilities for the different $k$ genetic groupings are shown in Table 1. The highest likelihood was observed for $k=3(\ln =-6161.7)$ and decreases for lower and higher $k$ values indicating that the data are consistent with of model of three genetically distinct groups. In terms of the genetic make-up of individual specimens (Fig. 2), some individuals were estimated to have a genetic make-up consisting $100 \%$ from a particular ancestral glacial source population whereas $34 \%$ of the individuals sampled were estimated to have a mixed ancestry. Individuals of mixed ancestry were present in most sky island populations (i.e. in 10 of the 14 populations). With the possible exception of the Absorka Mountains the absence of individuals of mixed ancestry most likely reflects limited sampling of individuals, especially in the Big Belt, Beaverhead, and Wind River Mountains.

The AMOVA detected significant population structuring of genomic variation at both the local and regional geographic scales (Table 2) corresponding to the recolonization of individual sky island populations and displacements into glacial refugia, respectively (Appendix). Regional and local geographic differentiation explained $15.1 \%$ and $13.5 \%$ of the total molecular variance, respectively. Similar but slightly higher values (see $\theta^{B}$ in Table 2 ) were obtained with the Bayesian approach that is not constrained by assumptions of Hardy-Weinberg equilibrium. Because estimates of inbreeding, $f$, are not reliable from dominant marker data, $\theta^{B}$ was estimated incorporating uncertainty about $f$. Comparison of the deviance information criterion 


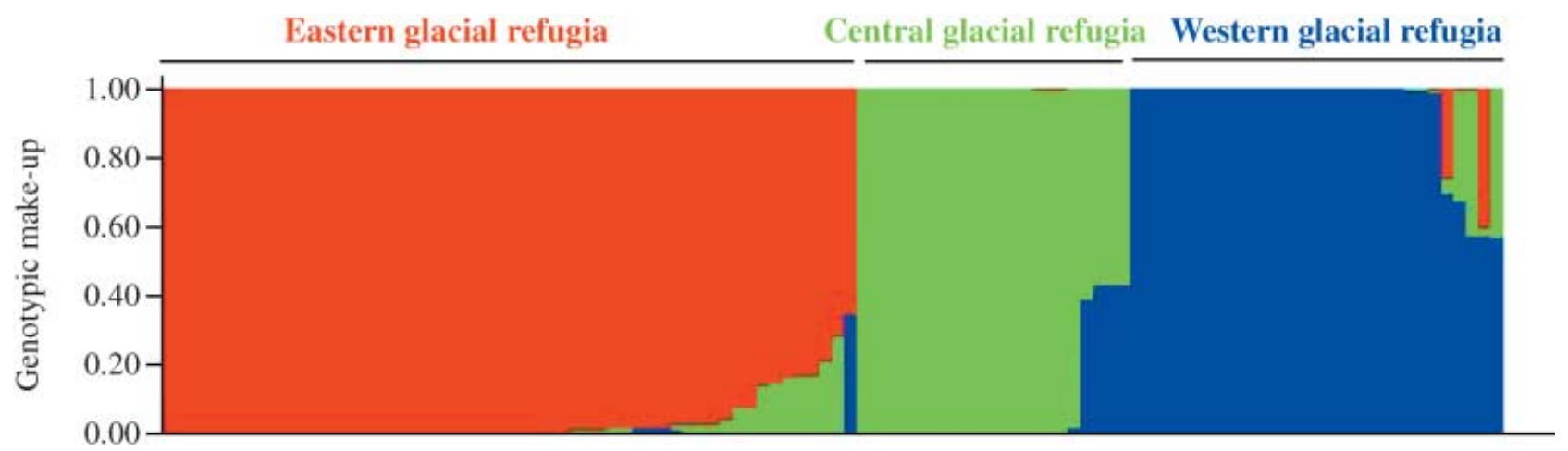

Fig. 2 Genotypic make-up for each individual from the three inferred glacial-source populations $(k=3)$ where each individual's genome is represented by a thin vertical line partitioned into coloured segments in proportion to the estimated ancestry. The genotypic composition of an eastern, central, and western ancestral glacial source population is represented in red, green, and blue, respectively. Results are shown for one run; each run of the algorithm yielded nearly identical results (burn-in 30000 iterations and collected data for 106 iterations for each run).

Table 2 Analysis of molecular variance (AMOvA) for grasshopper AFLP data*

\begin{tabular}{lrlrl}
\hline Source of variation & d.f. & S-statistict & $\%$ total & $P$ value \\
\hline Among glacial refugia & 2 & $\Phi_{\mathrm{CT}}=0.15$ & 15.1 & $<0.001$ \\
Sky island populations/glacial refugia & 11 & $\Phi_{\mathrm{SC}}=0.16$ & 13.5 & $<0.001$ \\
Individuals/populations & 120 & $\Phi_{\mathrm{ST}}=0.28$ & 71.4 & $<0.001$ \\
\hline
\end{tabular}

*Bayesian estimates (using HIскоRY: free model) where similar $\left(\theta^{B}=0.21\right.$ and 0.23$)$; $95 \%$ credible intervals overlap estimates from AMOvA (burn in 50000 and sampling run of 500000 iterations).

†CT, variance among groups of populations; SC, variance among the populations within groups; ST, variance among the individuals within a population.

(DIC) between each of the regional and local geographic structure models with a model of no structure (i.e. $\theta^{B}=0$; data not shown) provided further evidence that there are significant population genetic differences (e.g. Holsinger \& Wallace 2004), as predicted by analyses of mtDNA in Melanoplus oregonensis (Knowles 2001b).

Estimated genetic diversity $\left(H_{\mathrm{S}}\right)$ within individual M. oregonensis sky island populations ranged from 0.188 to 0.288 , with an average $H_{S}$ of 0.227 (Table 3). Levels of regional diversity corresponding to the different glacial ancestral source populations were similarly high (Nei 1987), with $H_{S}$ of $0.226,0.265$, and 0.251 for the populations from the eastern, western, and central parts of its range (see Appendix), respectively.

\section{Discussion}

Drift-induced divergence associated with the repeated shifts in species distributions and fragmentations of populations during the Pleistocene appears to have played an important role in the divergence of Melanoplus oregonensis. Despite the frequent shifts in species distributions and the larger effective population size of nuclear markers relative to mtDNA, the genetic signature of past demographic events
Table 3 Bayesian estimates of genetic diversity, $H_{\mathrm{S}}$ (defined as average panmictic heterozygosity within each population, Holsinger 1999; HICKORY, Holsinger et al. 2002)

\begin{tabular}{lll}
\hline Population & $H_{\mathrm{S}}$ & $95 \%$ credible intervals \\
\hline Absorka Mountains & 0.238 & $0.215-0.262$ \\
Maddison Mountains & 0.241 & $0.220-0.263$ \\
Big Snowy Mountains & 0.203 & $0.176-0.233$ \\
Gallatin Mountains & 0.251 & $0.226-0.278$ \\
Boulder Mountains & 0.227 & $0.194-0.263$ \\
Crazy Mountains & 0.222 & $0.197-0.249$ \\
Big Belt Mountains & 0.207 & $0.175-0.242$ \\
Gravelly Mountains & 0.219 & $0.196-0.244$ \\
Livingston Mountains & 0.188 & $0.157-0.222$ \\
Mission Mountains & 0.219 & $0.189-0.251$ \\
Beaverhead Mountains & 0.288 & $0.269-0.307$ \\
Tobacco Root Mountains & 0.229 & $0.207-0.253$ \\
Wind River Mountains & 0.227 & $0.199-0.256$ \\
Little Belt Mountains & 0.220 & $0.184-0.259$ \\
\hline
\end{tabular}

suggested by analysis of mtDNA sequences (Knowles 2001b) was also recovered from genomic patterns of variation. This correspondence is testament to the evolutionary significance of genetic drift in species divergence 
(Knowles 2004), even in the absence of long-term geographic barriers.

The effects of drift-induced species divergence are evident at two temporal and geographic scales corresponding to divergence associated with displacements into glacial refugia and recolonization of previously glaciated areas. Contrary to other recently expanded populations (e.g. Zink \& Dittmann 1993; Avise 1994), the results in this study indicate that the Plesistocene glacial cycles themselves played a direct role in species divergence (e.g. Comes \& Kadereit 2003; Ayoub \& Riechert 2004; Galbreath \& Cook 2004; Schönswetter et al. 2004; Yeh et al. 2004). The repartitioning of ancestral variation evident in this study without the wholesale purging of genetic variation (Fig. 2) also has intriguing implications for a potential interplay between selection and drift-induced divergence associated with biogeographic and demographic events (see below).

\section{Phylogeographic concordance}

The displacements into allopatric glacial refugia and recolonization of previously glaciated areas was postulated to be important in driving divergence in these grasshoppers (Knowles 2001a, b) and in promoting the radiation of the genus Melanoplus (Knowles et al. 1999; Knowles \& Otte 2000). However, as with many other studies, the species history was inferred from mtDNA. The actual evolutionary significance of past demographic and biogeographic events thereby remained unknown without assessing their impact on patterns of genomic variation, in addition to risk of misinterpreting the biogeographic and demographic past (Hey \& Machado 2003; Knowles 2004) as single loci may not accurately reflect the species history due to the stochastic nature of the lineage sorting process (Takahata 1989; Maddison 1997; Edwards \& Beerli 2000; Knowles \& Maddison 2002).

Tests of genomic differentiation indicate that both displacements into glacial refugia and recolonization of contemporary sky island populations contributed to divergence in M. oregonensis. This conclusion is robust and consistent across different analytical methodologies (Table 2), thereby confirming that historical inferences based on mtDNA were indeed accurate. There is substantial variation among the $F_{\mathrm{ST}}$ values of individual pairs of populations across all geographic distances (paralleling the patterns of differentiation observed in the mitochondrial DNA, Knowles 2001b), which is consistent with a drift-dominated system (Hutchinson \& Templeton 2000). This is in accord with the natural history of $M$. oregonensis, which is flightless and restricted to isolated montane meadows.

In addition to indicating that the historical inferences based on the mtDNA were accurate, the data also demonstrate the evolutionary significance of genetic drift associated with past biogeographic and demographic events.
Detecting the genealogical signature of such events was challenging even with mtDNA - there was considerable incomplete lineage sorting of mtDNA lineages at both local and regional geographic scales. Only with a geographically explicit coalescent model was historical divergence associated with displacements into allopatric refugia statistically discernable (Knowles 2001b). Consequently, during the dynamic Pleistocene, genomic patterns of differentiation (with the larger effective population size of nuclear loci) are only likely when genetic drift associated with past demographic events is considerable (Knowles 2004).

\section{Role of genetic drift in Pleistocene divergence}

The significant genetic differentiation (Table 2) without significant reductions in genetic diversity (Table 3) indicates that divergence reflects the repartitioning of ancestral variation. Not only was the genetic diversity within populations not very low (see Nei 1987 for comparative data), but many individuals also were of mixed ancestry (Fig. 2). Again, this pattern of significant genetic differentiation without purging ancestral variation corresponds to analyses of mtDNA sequences (Knowles 2001a, b).

This type of drift-induced divergence, in contrast to differentiation arising from severe bottlenecks, has two interesting implications. First, initial drift-induced differences may be further amplified by selection since the requisite variation for selection to act upon would be readily available. Divergence based on standing genetic variation can result in rapid adaptive evolution in contrast to divergence that relies on the appearance of new mutations (Orr \& Betancourt 2001; Gavrilets 2003). Second, the repartitioning of ancestral variation itself could set the stage for selection to operate. For example, changes in the shape of the adaptive landscape (e.g. Whitlock \& Fowler 1996; Knowles et al. 1999) or shifts of populations to different positions along the adaptive landscape (e.g. Gavrilets 1997, 2003) may result, potentially leading then to rapid selectively driven divergence.

In the context of $M$. oregonensis, and montane melanoplines in general, this potential interplay of drift-induced changes with selection might explain why these grasshoppers were able to speciate during the Pleistocene. The species are morphologically very similar, differing primarily in the shape of the male genitalia, a sexually selected character that may affect reproductive isolation (Eberhard 1996). If the phenotypic variation of such a character was affected like the genomic data, then the drift-induced changes may set the stage for rapid selectively driven divergence, for example, by initiating a runaway selective process. The male genitalia in Melanoplus species have undergone rapid divergence, as inferred from phylogenetic analysis (Knowles 2000). Moreover, because divergence is 
hierarchically structured (i.e. older divergence associated with displacements in glacial refugia and recent divergence associated with postglacial recolonization of the sky islands), reinforcing selection could also contribute to species divergence. Irrespective of whether divergence affecting reproductive characters is driven by drift or selection, when divergent sky island populations are displaced into a common glacial refuge, hybrids from such interpopulational matings may be at a selective disadvantage. Analysis of a closely related melanopline, Barytettix, has demonstrated that there is strong selection against hybrid males with intermediate genitalic morphologies (Knowles et al. 2005). Future studies will investigate whether there is geographic variation in genitalic characters in M. oregonensis to evaluate whether the proposed integrative model of drift-induced change and selectively driven divergence is tenable.

\section{Conclusions}

This study demonstrates that drift-induced divergence associated with both the colonization of sky islands and displacement into multiple glacial refugia during the Pleistocene has contributed to divergence in Melanoplus oregonensis. Significant genomic differentiation occurred in the face of frequent shifts in species distributions and was evident despite the larger effective population size of nuclear loci. The results clearly indicate that the historical biogeographic and demographic events during the Pleistocene are indeed of significant evolutionary consequence, and thereby demonstrate the important role genetic drift plays in species divergence, even in the absence of long-standing geographic barriers. Moreover, differentiation occurred via the repartitioning of ancestral variation, rather than by severe bottlenecks. This mode of divergence provides conditions in which initial drift-induced differences may be further amplified by selectively driven ones, generating the potential for rapid evolutionary divergence, and possibly explaining why speciation was not inhibited in these grasshoppers during the Pleistocene. When the results from this study are considered in conjunction with previous work on the timing of species divergence (Knowles 2000; Knowles \& Otte 2000 ), they support the contention that speciation did not simply coincide with the glaciations, but that the glaciations played an active role in diversification (Hewitt 1996; Weir \& Schluter 2004).

\section{Acknowledgements}

Thanks to members of the Knowles laboratory for their input, especially Ruben Omar Lastra for assisting with the manual gel runs. The research was funded by a National Science Foundation grant (DEB-04-47224) and a grant from the University of Michigan (Office of the Vice President for Research) to L.L.K.

\section{References}

Avise JC (1994) Molecular Markers, Natural History, and Evolution. Chapman \& Hall, London.

Avise JC, Walker D (1998) Pleistocene phylogeographic effects on avian populations and the speciation process. Proceedings of the Royal Society of London. Series B, Biological Sciences, 265, 457-463.

Ayoub NA, Riechert SE (2004) Molecular evidence for Pleistocene glacial cycles driving diversification of a North American desert spider, Agelenopsis aperta. Molecular Ecology, 13, 34533465.

Beaumont MA, Nichols RA (1996) Evaluating loci for use in the genetic analysis of population structure. Proceedings of the Royal Society of London. Series B, Biological Sciences, 263, 1619-1626.

Bermingham E, Rohwer S, Freeman S, Woods C (1992) Vicariance biogeography in the Pleistocene and speciation in North American wood warblers: a test of Mengel's model. Proceedings of the National Academy of Sciences, USA, 89, 6624-6628.

Bush MB (1994) Amazonian speciation: a necessarily complex model. Journal of Biogeography, 21, 5-17.

Carisio L, Cervella P, De Palestrini CI, Pero M, Rolando A (2004) Biogeographical patterns of genetic differentiation in dung beetles of the genus Trypocopris (Coleoptera, Geotrupidae) inferred from mtDNA and AFLP analyses. Journal of Biogeography, 31, 1149-1162.

Comes HP, Kadereit JW (2003) Spatial and temporal patterns in the evolution of the flora of the European Alpine system. Taxon, 52, 451-462.

Coyne JA, Orr HA (2004) Speciation. Sinauer Associates, Sunderland, Massachusetts.

Cracraft J, Prum RO (1988) Patterns and processes of diversification: speciation and historical congruence in some Neotropical birds. Evolution, 42, 603-620.

Eberhard WG (1996) Female Control: Sexual Selection by Cryptic Female Choice. Princeton University Press, Princeton, New Jersey.

Edwards SV, Beerli P (2000) Perspective: gene divergence, population divergence, and the variance in coalescence time in phylogeography studies. Evolution, 54, 1839-1854.

Elias SA (1996) The Ice-Age History of National Parks in the Rocky Mountains. Smithsonian Institution Press, Washington and London.

Excoffier L, Smouse PE, Quattro JM (1992) Analysis of molecular variance inferred from metric distances among DNA haplotypes: application to human mitochondrial DNA restriction data. Genetics, 131, 479-491.

Galbreath KE, Cook JA (2004) Genetic consequences of Pleistocene glaciations for the tundra vole (Microtus oeconomus) in Beringia. Molecular Ecology, 13, 135-148.

Gavrilets S (1997) Evolution and speciation on holey adaptive landscapes. Trends in Ecology \& Evolution, 12, 307-312.

Gavrilets S (2003) Models of speciation: what have we learned in 40 years? Evolution, 57, 2197-2215.

Green DM, Sharbel TF, Kearsley J, Kaiser H (1996) Postglacial range fluctuation, genetic subdivision and speciation in the western North American spotted frog complex, Rana pretiosa. Evolution, 50, 374-390.

Haffer J (1969) Speciation in Amazonian forest birds. Science, 165, 131-137.

Haffer J (1997) Alternative models of vertebrate speciation in Amazonia: an overview. Biodiversity and Conservation, 6, 451-476. 
Hawthorne DJ (2001) AFLP-based genetic linkage map of the Colorado potato beetle Leptinotarsa decemlineata: sex chromosomes and a pyrethroid-resistance candidate gene. Genetics, 158, $695-700$

Hewitt GM (1996) Some genetic consequences of ice ages, and their role in divergence and speciation. Biological Journal of the Linnean Society, 58, 247-276.

Hewitt GM (1999) Post-glacial re-colonization of European biota. Biological Journal of the Linnean Society, 68, 87-112.

Hey J, Machado CA (2003) The study of structured populations new hope for a difficult and divided science. Nature Reviews Genetics, 4, 535-543.

Hollin JT, Schilling DH (1981) Late Wisconsin-Weichselian mountain glaciers and small ice caps. In: The Last Great Ice Sheets (eds Denton GH, Hughes TJ), pp. 179-198. John Wiley \& Sons, New York.

Holsinger KE (1999) Analysis of genetic diversity in geographically structured populations: a Bayesian perspective. Hereditas, 130, 245-255.

Holsinger KE, Wallace LE (2004) Bayesian approaches for the analysis of population genetic structure: an example from Platanthera leucophaea (Orchidaceae). Molecular Ecology, 13, 887-894.

Holsinger KE, Lewis PO, Dey DK (2002) A Bayesian approach to inferring population structure from dominant markers. Molecular Ecology, 11, 1157-1164.

Hudson RR, Boos DD, Kaplan NL (1992) A statistical test for detecting geographic subdivision. Molecular Biology and Evolution, 9, 138-151.

Huntley B, Webb T III (1989) Migration: species' response to climatic variations caused by changes in the Earth's orbit. Journal of Biogeography, 16, 5-19.

Hutchinson DW, Templeton AR (2000) Correlation of pairwise genetic and geographic distance measures: inferring the relative influences of gene flow and drift on the distribution of genetic variability. Evolution, 53, 1889-1914.

Joseph L, Moritz C, Hugall A (1995) Molecular support for vicariance as a source of diversity in rainforest. Proceedings of the Royal Society of London. Series B, Biological Sciences, 260, 177-182.

Klicka J, Zink RM (1997) The importance of recent ice ages in speciation: a failed paradigm. Science, 277, 1666-1669.

Knowles LL (2000) Tests of Pleistocene speciation in montane grasshoppers from the sky islands of western North America (genus Melanoplus). Evolution, 54, 1337-1348.

Knowles LL (2001a) Genealogical portraits of speciation in montane grasshoppers (genus Melanoplus) from the sky islands of the Rocky Mountains. Proceedings of the Royal Society of London. Series B, Biological Sciences, 268, 319-324.

Knowles LL (2001b) Did the Pleistocene glaciations promote divergence? Tests of explicit refugial models in montane grasshoppers. Molecular Ecology, 10, 691-701.

Knowles LL (2004) The burgeoning field of statistical phylogeography. Journal of Evolutionary Biology, 17, 1-10.

Knowles LL, Maddison WP (2002) Statistical phylogeography. Molecular Ecology, 11, 2623-2635.

Knowles LL, Otte D (2000) Phylogenetic analysis of montane grasshoppers from western North America (genus Melanoplus, Acrididae: Melanoplinae). Annals of the Entomological Society of America, 93, 421-431.

Knowles LL, Coelho A, McNellis J, Greene KP, Futuyma DJ (1999) Tests of inbreeding effects on host shift potential in the phytophagous beetle Ophraella communa. Evolution, 53, 561-567.
Knowles LL, Chappell TM, Marquez EJ (2005) Concordance of hybrid clines reveals sexual selection's role in genitalic divergence and speciation. Evolution, in review.

Kocher TD, Lee WJ, Sobolewska H, Penman D, McAndrew B (1998) A genetic linkage map of a cichlid fish, the Tilapia (Oreochromis niloticus). Genetics, 148, 1225-1232.

Latta RG, Linhart YB, Mitton JB (2001) Cytonuclear disequilibrium and genetic drift in a natural population of ponderosa pine. Genetics, 158, 843-850.

Maddison W (1997) Gene trees in species trees. Systematic Biology, 46, 523-536.

Mayewski PA, Denton GH, Hughes TJ (1981) Late Wisconsin ice sheets in North America. In: The Last Great Ice Sheets (eds Denton GH, Hughes TJ), pp. 67-89. John Wiley \& Sons, New York.

Mengel RM (1964) The probable history of species formation in some northern wood warblers. Living Bird, 3, 9-43.

Mueller UG, Wolfenbarger L (1999) AFLP genotyping and fingerprinting. Trends in Ecology \& Evolution, 14, 389-394.

Nei M (1987) Molecular Evolutionary Genetics. Columbia University Press, New York.

Orr HA, Betancourt AJ (2001) Haldane's sieve and adaptation from the standing genetic variation. Genetics, 157, 875-884.

Parsons YM, Shaw KL (2002) Mapping unexplored genomes: a genetic linkage map of the Hawaiian cricket Laupala. Genetics, $162,1275-1282$.

Pielou EC (1991) After the Ice Age: The Return of Life to Glaciated North America. University of Chicago Press, Chicago.

Pritchard JK, Stephens M, Donnelly P (2000) Inferences of population structure using multilocus genotype data. Genetics, 155, 945-959.

Riddle BR (1996) The molecular phylogeographic bridge between deep and shallow history in continental biotas. Trends in Ecology E Evolution, 11, 187-228.

Roy K, Valentine JW, Jablonski D, Kidwell JM (1996) Scales of Climatic variability and time averaging in Pleistocene biotas: implications for ecology and evolution. Trends in Ecology \& Evolution, 11, 458-463.

Schönswetter P, Tribsch A, Niklfeld H (2004) Amplified fragment length polymorphism (AFLP) suggests old and recent immigration in the alps by the arctic-alpine annual Comastoma tenellum (Gentianaceae). Journal of Biogeography, 31, 1673-1681.

Singh G (1988) History of aridland vegetation and climate: a global perspective. Biological Review, 63, 159-195.

Takahata N (1989) Gene genealogy in three related populations: consistency probability between gene and population trees. Genetics, 122, 957-966.

Vekemans X, Beauwens T, Lemaire M, Roldán-Ruiz I (2002) Data from amplified fragment length polymorphism (AFLP) markers show indication of size homoplasy and of a relationship between degree of homoplasy and fragment size. Molecular Ecology, 11, 139-151.

Vos P, Hagers R, Bleeker M et al. (1995) AFLP: new technique for DNA fingerprinting. Nucleic Acids Research, 23, 4407-4414.

Wang Z, Baker AJ, Hill GE, Edwards SV (2003) Reconciling actual and inferred population histories in the house finch (carpodacus mexicanus) by AFLP analysis. Evolution, 57, 28522864.

Webb T III, Bartlein PJ (1992) Global changes during the last 3 million years, climatic controls and biotic responses. Annual Review of Ecology and Systematics, 23, 141-173. 
Weir JT, Schluter D (2004) Ice sheets promote speciation in boreal birds. Proceedings of the Royal Society of London. Series B, Biological Sciences, 271, 1881-1887.

Whitlock MC, Fowler K (1996) The distribution among populations in phenotypic variance with inbreeding. Evolution, 50, 1919-1926.

Wolfe JA (1987) An overview of the origins of the modern vegetation and flora of the northern Rocky Mountains. Annals of the Missouri Botanical Garden, 74, 785-803.

Yeh WB, Chang YL, Lin CH, Wu FS, Yang JT (2004) Genetic differentiation of Loxoblemmus appendicularis complex (Orthoptera: Gryllidae): speciation through vicariant and glaciation events. Annals of the Entomological Society of America, 97, 613-623.

Zink RM, Dittmann DL (1993) Gene flow, refugia, and evolution of geographic variation in the song sparrow (Melospiza melodia). Evolution, 47, 717-729.
This study complements my early work and provides much needed insights into what processes have been involved in the spectacular radiation of Melanoplus grasshoppers during the turbulent Pleistocene. My current research on Melanoplus involves additional genomic studies, which when combined with quantification of morphological rates of divergence, will be able to address the contributions of historical biogeographic and demographic factors, as well as selective forces in the speciation process. Corinne Richards is a PhD student whose research interests are in conservation biology, focusing specifically on integrative ecological and genetic approaches to studying the endangered Panamanian golden frogs in the genus Atelopus. 
4032 L. L. KNOWLES and C. L. RICHARDS

\section{Appendix}

Sampling localities with number of individuals of Melanoplus oregonensis sampled at each site $(n)$. The geographic positions of sky island populations are indicated in Fig. 1.

Sky island populations

$n$

Absorka Range, Beartooth Plateau, Carbon Co., MT

Madison Range, Lone Mountain, Madison Co., MT

Big Snowy Mountains, Fergus Co., MT

Gallatin Range, Teton Co., WY

Boulder Mountains, Jefferson Co., MT

Crazy Mountains, Sweet Grass Co., MT

Big Belt Mountains, Meagher Co., MT

Gravelly Range, Madison Co., MT

Livingston Range, Glacier Co., MT

Mission Range, Missoula Co., MT

Beaverhead Mountains, Sawtelle Peak, Fremont Co., ID

Tobacco Root Mountains, Madison Co., MT

Wind River Range, Teton Co., WY

Little Belt Mountains, Cascade Co., MT

Total

*Population assignments based on mitochondrial gene tree (see Knowles 2001b).
Ancestral refugial population*

Eastern

Western

Eastern

Western

Central

Eastern

Central

Western

Eastern

Central

Western

Western

Eastern

Western 